\title{
ON SOME QUANTUM AND ANALYTICAL PROPERTIES OF FRACTIONAL FOURIER TRANSFORMS
}

\author{
JIANHONG SHEN \\ School of Mathematics \\ University of Minnesota \\ Minneapolis, MN 55455, USA \\ E-mail: jhshen@math.umn.edu
}

\begin{abstract}
Fractional Fourier transforms (FrFT) are a natural one-parameter family of unitary transforms that have the ordinary Fourier transform embedded as a special case. In this paper, following the efforts of several authors, we explore the theory and applications of FrFT, from the standpoints of both quantum mechanics and analysis. These include the phase plane interpretation of FrFT, FrFT's role in the order reduction of certain classes of differential equations, the integral representation of FrFT, and its Paley-Wiener theorem and Heisenberg uncertainty principle. Our two major tools are quantum operator algebra and asymptotic analysis such as the singular perturbation theory and the stationary phase technique. ${ }^{\text {a }}$
\end{abstract}

aTo appear in Wavelet Analysis: Twenty Year's Developments, Ed. Z.-X. Zhou, World Scientific Press, Singapore, 2002.

\section{Introduction}

The concept of fractional Fourier transforms (FrFT) was first introduced by physicist Namias in $1980^{1}$, based on the spectral structure of the classical Fourier transform. It is well known that the eigenvalues of Fourier transform are $e^{i n \frac{\pi}{2}}, n=0,1, \cdots$, and the associated eigenvectors are the Hermite functions (i.e., the normalized Hermite polynomials weighted by the factor $e^{-\frac{x^{2}}{2}}$ ). Fourier transform is diagonalized by this complete set of eigen-functions. Namias was interested in those tranforms that commute with Fourier transform but still not too wild. As a result, he considered a general transform $\mathcal{F}_{\alpha}$ whose eigenvalues are given by $e^{i n \alpha}, n=0,1, \cdots$ and whose ordered eigen-functions are identical to those of Fourier tranform. For each real scalar $\alpha, \mathcal{F}_{\alpha}$ is called a fractional Fourier transform of order $\alpha$. Thus the classical Fourier transform is naturally embedded within the one-parameter family of FrFT's.

Namias ${ }^{1}$ was able to generalize many results of classical Fourier transform to FrFT, based on the properties of the Hermite orthogonal polynomials, such 
as the three-term recursion relation and the reproducing kernel formula. The successful application of FrFT in certain classes of evolutionary or stationary Schrödinger equations in quantum mechanics clearly demonstrated the power of this new tool. Though some of his derivations were in a formal level, Namias set up the right framework for further studies on FrFT.

The work was later supplemented by McBride and Kerr ${ }^{2}$, who developed the necessary mathematical foundation for FrFT. It was a pity, however, that some of the important motivations and intuitions from quantum mechanics were lost. As a result, many mathematical calculations became difficult to follow.

Recently Dattoli, Torre, and Mazzacurati ${ }^{3}$ re-emphasized the quantum mechanics origin of FrFT, by treating $\mathcal{F}_{\alpha}$ 's as the time evolution of a free harmonic oscillator, and interpreting $\alpha$ as the "time" parameter. Such physics point of view allowed them to benefit from the two different but equivalent pictures of Schrödinger and Heisenberg: the evolution of states and the evolution of observables. Furthermore, The authors ${ }^{3}$ were able to show that the family of FrFT is as natural as the classical Fourier transform, at least from the underlying physics point of view. The authors, however, did not seem to go far enough endowed with this new degree of freedom from physics.

In this paper, we attempt to further explore three aspects of FrFT:

(1) its quantum mechanics foundation,

(2) the general theory of order reduction of certain classes of differential equations by FrFT, and

(3) some important analytical properties generalized from Fourier transform, such as the Paley-Wiener theorem and the uncertainty principle.

Our major tools are quantum operator algebra, and asymptotic analysis based on the singular perturbation theory and the stationary phase technique ${ }^{4}$.

In Section 2, we introduce FrFT as the time evolution operator in quantum mechanics, following the approach of Dattoli et al. ${ }^{3}$. In Section 3 , the classical and quantum correspondence leads to the rotation interpretation of FrFT in the phase plane, by which the "time" parameter $\alpha$ becomes the angle of a phase rotation. Such a picture also allows us to connect FrFT to the two most important operators in the quantum theory of harmonic oscillators - the raising and lowering operators $g_{+}$and $g_{-}$.

Section 4 and 5 explore the mathematical theory of order reduction of differential equations based on FrFT, as first mentioned in ${ }^{1,2}$. In Section 4, the difference between the classical Fourier transform and FrFT is explained by the position-momentum coupling and decoupling mechanisms, which play an important role for the order reduction of differential equations. A general result 
on order reduction is established. In Section 5, by the singular perturbation theory in asymptotic analysis, we are able to recapture and "zoom into" the lost order reduced by FrFT.

Section 6 discusses the integral representation of FrFT and some important issues that have been missing in the literature. The quadratic phase associated with an FrFT activates the stationary phase method in asymptotic analysis, and leads to an asymptotic formula for small $\alpha$ 's. We also extend two important results of Fourier transform to FrFT: the Paley-Wiener theorem and the analogy of the celebrated Heisenberg uncertainty principle. Efficient numerical implementation based on Fast Fourier Transform (FFT) is also briefly discussed in the end.

\section{Quantization and Periodicity}

It has been a familiar fact in classical Fourier analysis (and digital signal processing) that uniformly discretizing (or sampling) a function of position is equivalent to periodizing its Fourier transform in the momentum space. While this correspondence is crystal clear in the context of position and momentum, it has gained less attention in the duality of time and energy.

In terms of time, quantum mechanics observes two equivalent pictures of the evolutionary world, namely, the Schrödinger picture in which a state evolves according to the celebrated Schrödinger equation, and the Heisenberg picture in which not the state, but the observables (Hermitian operators) evolve. Connecting the two is the unitary time evolution operator $U_{t}=e^{i t H}$, where $H$ denotes the Hamiltonian of the system, and the Planck's constant $\hbar$ has been set to 1 for convenience. $U_{t}$ can be symbolically understood as the Fourier transform of energy. It is therefore expected that the duality of discretization-perodization mentioned above should find its parallelism here. In fact, in the time-energy context, it is better to be described as the quantizationperiodization duality. Quantization is the physics way of discretization.

Definition (Integral Observables). An observable $A$ in a quantum system is said to be integral if there exist constants $a_{0}$ and $a_{1}$ so that for any observation (i.e. eigenvalue) $a$ of $A,\left(a-a_{0}\right) / a_{1}$ is an integer.

The Hamiltonian of a harmonic oscillator and the $z$-component angular momentum $J_{z}$ are the two most well known integral observables. Notice that by this definition, it is unnecessary for the observations being uniformly distributed (or quantized).

Let $\alpha$ denote the real scalar variable dual to an integral observable $A$. Then

$$
A_{\alpha}=e^{i \alpha\left(A-a_{0}\right)}
$$


defines a one-parameter family of unitary operators, where $a_{0}$ is as in the definition. Since $A$ is integral, $A_{\alpha}$ is in fact periodic with respect to $\alpha$.

FrFT is directly based on the Hamiltonian of the harmonic oscillator

$$
H_{0}=-\frac{1}{2} \frac{d^{2}}{d x^{2}}+\frac{1}{2} x^{2}
$$

The energy spectra of a harmonic oscillator consist of all positive half integers $\left\{\frac{1}{2}, \frac{3}{2}, \cdots\right\}$. Hence $H_{0}$ is an integral observable. Define $H=H_{0}-\frac{1}{2}$, so that the spectra are shifted downward to be all nonnegative integers. Then, an FrFT associated to a real scalar $\alpha$ is defined as $\mathcal{F}_{\alpha}=e^{i \alpha H}$.

Proposition 2.1. $\mathcal{F}_{\alpha}$ is $2 \pi$-periodic with respect to $\alpha$, and

$$
\mathcal{F}_{\alpha}=\sum_{n=0}^{\infty} e^{i n \alpha}|n\rangle\langle n|,
$$

where the ket $|n\rangle$ denotes the $n^{\text {th }} L^{2}$-normalized Hermite function, and the bra $\langle n|$ its transpose.

This was the original formula of FrFT by Namias ${ }^{1}$. The evolutionary point of view we have discussed was first propsed by Dattoli et al. in ${ }^{3}$. It is easy to see that the classical Fourier transform (noticing that we use $e^{i x y}$ instead of $\left.e^{-i x y}\right)$

$$
\mathcal{F} f(x)=\frac{1}{\sqrt{2 \pi}} \int_{-\infty}^{\infty} f(y) e^{i x y} d y
$$

is a special FrFT that corresponds to $\alpha=\frac{\pi}{2}$, since

$$
\mathcal{F}|n\rangle=i^{n}|n\rangle, \quad n=0,1, \cdots .
$$

The wide applicability of the classical Fourier transform in science and engineering raises many questions in terms of FrFT. For example, what is so special about $\alpha=\pi / 2$ ? or what is the real advantage for $\alpha$ being $\pi / 2$ ? and can we generalize most results of classical Fourier transform to FrFT? This paper intends to give some answers. Since $\mathcal{F}_{\alpha}=\mathcal{F}^{m} \circ \mathcal{F}_{\alpha-m \pi / 2}, m=0,1,2,3$, from now on, we shall assume that $\alpha \in[0, \pi / 2]$.

\section{Rotation, Lowering, and Raising}

Let $p=-i d / d x$ denote the momentum operator, and $q=(x, p)^{\mathrm{T}}$ the vector operator corresponding to a phase point in classical mechanics. In addition, denote by $R_{\alpha}$ the rotation matrix in the phase plane:

$$
\left[\begin{array}{cc}
\cos \alpha & \sin \alpha \\
-\sin \alpha & \cos \alpha
\end{array}\right] .
$$


Then one of most important results by Dattoli et al. ${ }^{3}$, McBride and Kerr ${ }^{2}$, and Namias ${ }^{1}$ can be reorganized in a neat way.

Proposition 3.1. For any real parameter $\alpha, \mathcal{F}_{\alpha} q \mathcal{F}_{\alpha}^{\dagger}=R_{\alpha} q$.

It gives, in the Heisenberg picture, the "time" evolution of the phase operator $q$ in a system described by the shifted Hamiltonian $H$ of a harmonic oscillator. Due to the quantization of energy, the "time" parameter $\alpha$ becomes periodic, and by the Proposition indeed it can be interpreted as the angle of a rotation. This probably explains that $\alpha$ has rarely been replaced by the symbol $t$ (for time) in the literature.

In the ordinary Fourier transform, the angle $\alpha=\pi / 2$. Thus $x$ and $p$ are still decoupled after transform: $x$ is tranformed (or "rotated") to $p$, while $p$ to $-x$. For a general FrFT, $x$ and $p$ become coupled. We are therefore led to the concept of eigenphases.

Definition (Eigenphase). A nonzero operator $g=a x+b p$ for some complex scalars $a$ and $b$ is called the eigenphase of $\mathcal{F}_{\alpha}$, if $\mathcal{F}_{\alpha} g \mathcal{F}_{\alpha}^{\dagger}=\lambda g$, for some complex scalar $\lambda$.

Theorem 3.1. Define

$$
g_{-}=x+i p=x+D \quad \text { and } \quad g_{+}=x-i p=x-D .
$$

Then up to a multiplicative scalar, these are the only two eigenphases of $\mathcal{F}_{\alpha}$.

Proof. By Proposition 3.1, $g=a x+b p$ is an eigenphase if and only if $(a, b)$ is a left eigenvector of the rotation matrix $R_{\alpha}$. And the $\lambda$ in the above definition precisely corresponds to the eigenvalue of $R_{\alpha}$. From linear algebra, the two eigenpairs of $R_{\alpha}$ are: $(1,-i)$ for $\lambda=e^{i \alpha}$, and $(1, i)$ for $\lambda=e^{-i \alpha}$. Hence the theorem follows.

In other words, we have

$$
\mathcal{F}_{\alpha} g_{-} \mathcal{F}_{\alpha}^{\dagger}=e^{-i \alpha} g_{-} \quad \text { and } \quad \mathcal{F}_{\alpha} g_{+} \mathcal{F}_{\alpha}^{\dagger}=e^{i \alpha} g_{+} \cdot
$$

But only one is independent since $g_{-}^{\dagger}=g_{+}$. Taking derivative with respect to $\alpha$ and evaluating at $\alpha=0$, we obtain

$$
\left[H, g_{+}\right]=g_{+} \quad \text { and } \quad\left[H, g_{-}\right]=-g_{-} .
$$

These are the two familiar brackets in quantum mechanics, from which, the spectral analysis for the harmonic oscillator can be carried out purely algebraically. $g_{+}$and $g_{-}$are like the eigenvectors of $H$ in the bracket algebra. 
Physicists call them the raising and lowering operators, since they shift the spectra of $H$ by

$$
g_{-}|n\rangle=c_{n}^{-}|n-1\rangle \quad \text { and } \quad g_{+}|n\rangle=c_{n}^{+}|n+1\rangle,
$$

for some real scalars $c_{n}^{ \pm}, n=0,1, \cdots$. Here we have defined $|-1\rangle=0$.

Proposition 3.1 (or equivalently, Eq. (1)) is the physics that determines mathematically the transform of $x$ and $d / d x$ under FrFT. Most of the operator calculus carried out in ${ }^{3,2,1}$ can be easily derived from it.

\section{Reduction of Orders by FrFT}

Why has Fourier transform been so powerful a tool in analysis? It is mostly because that when $\alpha=\pi / 2, x$ and $p$ (or $D$ ) are completely decoupled. Therefore, under Fourier transform, a differential operator with constant coefficients in the form of

$$
L=D^{n}+a_{1} D^{n-1}+a_{2} D^{n-2}+\cdots
$$

can be simplified to a pure multiplier

$$
\mathcal{F} L \mathcal{F}^{\dagger}=(-i x)^{n}+a_{1}(-i x)^{n-1}+a_{2}(-i x)^{n-2}+\cdots .
$$

However, this advantage turns to a disadvantage for operators like

$$
L=D^{2}+x^{2} \quad \text { or } \quad M=D^{2}-i x D+x^{2},
$$

in which the coefficients are polynomials of $x$. Direct Fourier transform contributes almost nothing since

$$
-\mathcal{F} L \mathcal{F}^{\dagger}=x^{2}+D^{2}=L \quad \text { and } \quad-\mathcal{F} M \mathcal{F}^{\dagger}=M-i .
$$

The order has not been reduced at all. This is mainly because that $x$ and $D$ are decoupled under Fourier transform, and do not communicate and coordinate to each other to achieve the goal of order reduction.

In contrast, FrFT can be expected to resolve the issue thanks to its coupling of $x$ and $D$. We shall first proceed with a typical example, whose formal form first appeared in Namias' original work ${ }^{1}$, and was made rigorous in ${ }^{2}$. Then a general result will be established in the end.

For example, let us try to find a special solution to

$$
u_{x x}(x)+x^{2} u(x)=0, \quad x \in \mathbb{R} .
$$

(The exact solutions can be explicitly expressed ${ }^{4}$ by Bessel functions $J_{\nu}$ ). Notice that once a special solution has been obtained, the classical method of order reduction in the ODE theory can help find another linearly independent one easily. 
The associated differential operator is $L=D^{2}+x^{2}$, or in terms of the momentum operator $p, L=x^{2}-p^{2}$. Therefore, under an FrFT $\mathcal{F}_{\alpha}, L$ is transformed to

$$
\begin{aligned}
\hat{L} & =(x \cos \alpha+p \sin \alpha)^{2}-(-x \sin \alpha+p \cos \alpha)^{2} \\
& =-\cos (2 \alpha) p^{2}+\cos (2 \alpha) x^{2}+2 \sin (2 \alpha) x p-i \sin (2 \alpha) .
\end{aligned}
$$

Notice that we have applied the commutator $[p, x]=-i$. Now if we choose $\alpha=\pi / 4$, the original second order equation becomes a first order one:

$$
2 x \hat{u}_{x}(x)+\hat{u}(x)=0,
$$

of which the general solution is easily to be worked out: $\hat{u}=C / \sqrt{|x|}$. Its inverse transform leads to one special class of solutions to the original second order equation:

$$
u=C \sqrt{|x|} J_{-1 / 4}\left(\frac{x^{2}}{2}\right) .
$$

More generally, let $L$ be an element in the non-commutative ring $\mathbb{C}[x, D]$; that is, a differential operator with polynomial coefficients.

Definition (D-Dominant and Leading Term). Suppose

$$
L=p_{0}(x) D^{n}+p_{1}(x) D^{n-1}+\cdots, \quad p_{k}(x) \in \mathbb{C}[x], p_{0} \neq 0 .
$$

We say that $L$ is $D$-dominant if for $k=0,1, \cdots, n, \operatorname{deg} p_{k} \leq k$. Assuming that

$$
p_{k}(x)=a_{k} x^{k}+a_{k, k-1} x^{k-1}+a_{k, k-2} x^{k-2}+\cdots .
$$

Then the operator

$$
L_{0}=a_{0} D^{n}+a_{1} x D^{n-1}+a_{2} x^{2} D^{n-2}+\cdots
$$

is called the leading term of $L$.

The leading symbol of a D-dominant operator $L$ of order $n$ is the following homogeneous polynomial in the classical phase plane $(x, p)$ (that is, $x$ and $p$ are scalars, not operators):

$$
\zeta(x, p)=a_{0} i^{n} p^{n}+a_{1} i^{n-1} x p^{n-1}+a_{2} i^{n-2} x^{2} p^{n-2}+\cdots,
$$

provided that the leading term of $L$ is given as in the definition.

Theorem 4.1. (Reduction of Order) Let $\zeta(x, p)$ be the leading symbol of a $D$-dominant differential operator L. If $\zeta(x, p)$ has at least one zero in the real projection line $\mathbb{R P}^{1}$, then there exists an angle $\alpha$ whose associated FrFT $\mathcal{F}_{\alpha}$ reduces one order of $L$. 
Proof. It is clear that $L_{0}$ determines the order of $\hat{L}=\mathcal{F}_{\alpha} L \mathcal{F}_{\alpha}^{\dagger}$ completely, since $L$ is D-dominant. On the other hand, just as shown in the example, the coefficient attached to $D^{n}$ in $\hat{L_{0}}$ is exactly $(-i)^{n} \zeta(\sin \alpha, \cos \alpha)$. Since $\zeta(x, p)$ contains at least one zero in $\mathbb{R P}^{1}$, one can find an angle $\alpha$, such that $\zeta(\sin \alpha, \cos \alpha)=0$. The FrFT associated to this $\alpha$ therefore reduces at least one order of $L$.

We conclude this section by an example. Consider the $4^{\text {th }}$ order differential equation

$$
u_{x x x x}+4 x^{2} u_{x x}+3 x^{4} u=0 .
$$

Let $L$ be the associated differential operator. It is easy to check that $L$ is indeed $\mathrm{D}$-dominant. The leading symbol is given by

$$
\zeta(x, p)=(i p)^{4}+4 x^{2}(i p)^{2}+3 x^{4}=p^{4}+3 x^{4}-4 x^{2} p^{2} .
$$

By setting $\zeta(x, p)=0$, we obtain in fact four angles whose associated $\mathcal{F}_{\alpha}$ 's all can reduce the equation to a $3^{\text {rd }}$ one:

$$
\alpha_{1,2}= \pm \frac{\pi}{4} \quad \text { and } \quad \alpha_{3,4}= \pm \frac{\pi}{6} .
$$

In the coming section, we shall see that these angles do reveal some important information about the solution space of the differential equation.

\section{Reduction of Orders - Singular Perturbation Theory}

In this section, by applying the singular perturbation theory in asymptotic analysis, we attempt to "zoom into" the detailed process of order reduction by FrFT.

Let us revisit the example $u_{x x}+x^{2} u=0$. We shall study its behavior under $\mathcal{F}_{\alpha}$ when $\alpha$ is near the critical angle $\pi / 4$. The analysis presented below applies to more general situations as well.

Set $\alpha=\pi / 4-\epsilon / 2$ with $\epsilon \ll 1$. Then $\cos (2 \alpha)=\sin \epsilon$ and $\sin (2 \alpha)=\cos \epsilon$. Define $\hat{u}^{\epsilon}=\mathcal{F}_{\alpha} u$. To the first order, the original equation is transformed to

$$
\epsilon\left(\hat{u}_{x x}^{\epsilon}+x^{2} \hat{u}^{\epsilon}\right)-i\left(x \hat{u}_{x}^{\epsilon}+\hat{u}^{\epsilon}\right)=0
$$

which is a singular perturbation problem with respect to $\epsilon$. The so called slowly varying solution ${ }^{4}$ is obtained by setting $x \hat{u}_{x}^{\epsilon}+\hat{u}^{\epsilon}=0$, which is exactly the equation we have discussed in the previous section.

Let us track down the other "lost" linearly independent solutions $\hat{u}$. The homogeneity of the equation allows us to assume that $\hat{u}$ is of order $O(1)$. 
Introducing the fast variable $y=x / \delta$, and $\hat{u}(y)=\hat{u}^{\epsilon}(x)$, with some small zooming scale $\delta$ to be determined later. Then Eq.(2) becomes

$$
\frac{\epsilon}{\delta^{2}} \hat{u}_{y y}+\epsilon \delta^{2} y^{2} \hat{u}-i\left(2 y \hat{u}_{y}+\hat{u}\right)=0 .
$$

A balance of dominant terms ${ }^{4}$ implies that $\delta=\sqrt{\epsilon}$ (assuming that $\epsilon>0$ ) and the equation for the so called rapidly varying solution is

$$
\hat{u}_{y y}-i\left(2 y \hat{u}_{y}+\hat{u}\right)=0 .
$$

This new equation can be easily solved by the ordinary Fourier transform, and one special solution is given by

$$
\hat{u}(y)=C \sqrt{|y|} \exp \left(i \frac{y^{2}}{2}\right) J_{-\frac{1}{4}}\left(\frac{y^{2}}{2}\right) .
$$

By the power series expression ${ }^{4}$ of $J_{\nu}$ at $y=0$, it is clear that $\hat{u}(y)$ is continuous at 0 and nonzero. The asymptotic formula ${ }^{4}$ of $J_{\nu}(y)$ for large $y$ also shows that $\hat{u}(y)$ is of order $O\left(|y|^{-1 / 2}\right)$. Our assumption that $\hat{u}$ is of order $O(1)$ is indeed valid.

Therefore, the singular solution to $(2)$ is $\hat{u}^{\epsilon}(x)=\hat{u}(x / \sqrt{\epsilon})$. In particular, we have found another approximate solution to the original equation, independent of the one discussed in the previous section:

$$
u^{\epsilon}(x)=\mathcal{F}_{\pi / 4-\epsilon / 2}^{-1} \hat{u}^{\epsilon}=\mathcal{F}_{-\pi / 4+\epsilon / 2} \hat{u}^{\epsilon} \simeq \mathcal{F}_{-\pi / 4} \hat{u}^{\epsilon} .
$$

The last step is due to that $\mathcal{F}_{\alpha}$ depends smoothly on $\alpha$ for $0<\alpha<\pi$ (see the integral representation in the next section).

This is the solution that has been lost when $\mathcal{F}_{\pi / 4}$ is directly applied to the original equation $u_{x x}+x^{2} u=0$. We call such loss the resonance phenomenon for the following reason.

First redefine $\hat{u}^{\epsilon}(x)$ by adding a normalization multiplier

$$
\hat{u}^{\epsilon}(x)=\frac{c}{\sqrt{\epsilon}} \hat{u}\left(\frac{x}{\sqrt{\epsilon}}\right)
$$

so that $\hat{u}^{\epsilon}(x)$ converges to the Dirac $\delta$-function as $\epsilon \rightarrow 0$ (since the equation is homogeneous). Corresponding to this new solution, the inverse $\operatorname{FrFT} u^{\epsilon}(x) \simeq$ $\mathcal{F}_{\pi / 4}^{-1} \hat{u}^{\epsilon}$ shall converge to $u^{*}=\mathcal{F}_{-\pi / 4} \delta$ in the asymptotic sense. From Namias' integral representation in the coming section, we have

$$
u^{*}(x)=\text { const. } \exp \left(i \frac{x^{2}}{2}\right)
$$

which is indeed the approximate solution to the original equation for $x \gg 1$ since

$$
u_{x x}^{*}+x^{2} u^{*}=i u^{*} .
$$


For large $x$, the term $i u^{*}$ is negligibly small compared with the other two. We must point out that in asymptotic analysis ${ }^{4}$, the leading term of the solution for large $x$ contains the factor $1 / \sqrt{|x|}$. Our approximate solution $u^{*}$ has been obtained from the singular perturbation in the FrFT domain, and this factor has been lost. However, the phase factors are identical. (In physics, the phase is the most crucial information for the resonance phenomenon.)

It is in this sense that the approximate solution $u^{*}$ is said to be resonant with $\mathcal{F}_{\pi / 4}$. What happens here can be explained in a more general picture as follows. There exists one direction in the solution space of the original equation, whose phase factor is exactly the conjugate of the quadratic phase factor (see the integral formula in the next section) in $\mathcal{F}_{\alpha}$. This direction is "lost" under $\mathcal{F}_{\alpha}$ because its image blows up (or resonates) to become the Dirac- $\delta$ function.

\section{The Integral Representation and Properties}

In this section, we discuss the integral representation of FrFT and its properties. The quadratic phase factor allows the application of the stationary phase method for establishing an asymptotic formula for small angles $\alpha$. We also extend the classical Paley-Wiener theorem and Heisenberg uncertainty principle to FrFT's. Efficient computational implementation based on FFT is briefly discussed in the end.

First define two unitary operators in $L^{2}(\mathbb{R})$ :

$$
g_{a} f(x):=\exp \left(-i \frac{a x^{2}}{2}\right) f(x) \text { and } \quad s_{\lambda} f(x):=\sqrt[4]{\lambda} f(\sqrt{\lambda} x) .
$$

$g_{a}$ is a pure phase multiplier while $s_{\lambda}$ is an ordinary scaling operator. We assume that both $a$ and $\lambda$ are positive. Further define $G_{a, \lambda}:=g_{a} \circ s_{\lambda}$, and

$$
T_{a, \lambda}:=G_{-a, \lambda}^{\dagger} \circ \mathcal{F} \circ G_{a, \lambda}=s_{\lambda}^{-1} \circ g_{a} \circ \mathcal{F} \circ g_{a} \circ s_{\lambda},
$$

where $\mathcal{F}$ denotes Fourier transform. Each $T_{a, \lambda}$ is unitary and its complete integral formula can be easily worked out:

Proposition 6.1. (Integral Representation)

$$
T_{a, \lambda} f(x)=\frac{1}{\sqrt{2 \pi \lambda}} \exp \left(-i \frac{a x^{2}}{2 \lambda}\right) \int_{-\infty}^{\infty} d y f(y) \exp \left(-i \frac{a y^{2}}{2 \lambda}+i \frac{x y}{\lambda}\right) .
$$

Now $T_{a, \lambda}$ gives the integral representation of $\mathcal{F}_{\alpha}$.

Theorem 6.1. (Namias' Theorem) For all $\alpha \in\left(0, \frac{\pi}{2}\right]$,

$$
\mathcal{F}_{\alpha}=e^{i \frac{1}{2}\left(\frac{\pi}{2}-\alpha\right)} \cdot T_{\cos \alpha, \sin \alpha} .
$$


Proof. Namias' proof follows immediately from Mehler's formula ${ }^{5}$ on Hermite functions:

$\exp \left(\frac{x^{2}+y^{2}}{2}\right) \sum_{n=0}^{\infty} e^{i n \alpha}|n\rangle(x)\langle n|(y)=\frac{1}{\sqrt{1-e^{2 i \alpha}}} \exp \left(\frac{2 x y e^{i \alpha}-\left(x^{2}+y^{2}\right) e^{2 i \alpha}}{1-e^{2 i \alpha}}\right)$,

where as before $|n\rangle(x)$ denotes the $n^{\text {th }}$ normalized Hermite function.

The nontrivial quadratic phase factor $g_{\alpha}($ with $\alpha \neq 0)$ allows $\mathcal{F}_{\alpha}$ to have a larger definition domain than Fourier transform. That is, rapid phase cancellation associated with the quadratic phase allows the function to be transformed to blow up at $\infty$ at some mild rate. For example, based on Abel's summation theorem, we can easily establish the following result.

Proposition 6.2. $\alpha \in\left(0, \frac{\pi}{2}\right)$. Then for all $\gamma \in(-1,1), \mathcal{F}_{\alpha}|x|^{\gamma}$ is well defined.

Furthermore, we can apply the technique of stationary phase in asymptotic analysis ${ }^{4}$ to the quadratic phase to establish asymptotic approximations.

Theorem 6.2. (Stationary Phase) Suppose $A=a / \lambda \gg 1$, and that $f(x)$ satisfies the regularity conditions for the stationary phase approximation ${ }^{4}$. Then we have the asymptotic (with respect to A) leading term equivalence

$$
\mathcal{F} \circ G_{a, \lambda} f(x) \simeq \frac{e^{i\left(\frac{x^{2}}{2 a}-\frac{\pi}{4}\right)}}{\sqrt{A \sqrt{\lambda}}} f\left(\frac{x}{A \sqrt{\lambda}}\right) .
$$

Applying this theorem to $\mathcal{F}_{\alpha}$ when $\alpha \ll 1$, and noticing that $a=\cos \alpha \simeq 1$ and $\lambda=\sin \alpha \simeq \alpha$, we have

Proposition 6.3. (Small $\alpha$ ) If $\alpha \ll 1$, the leading term of $\mathcal{F}_{\alpha} f(x)$ is

$$
\mathcal{F}_{\alpha} f(x) \simeq \exp \left(i \frac{\alpha\left(x^{2}+1\right)}{2}\right) f(x) .
$$

The integral representation and the structure of $T_{a, \lambda}$ make it possible to generalize many results in Fourier analysis to FrFT. Some have already appeared in ${ }^{3,2,1}$. Here we first entend the classical Paley-Wiener theorem in Fourier analysis.

Theorem 6.3. (Paley-Wiener for FrFT's) Fix an angle $\alpha \in\left(0, \frac{\pi}{2}\right]$. Let $\hat{f}(z)$ denote the $\mathcal{F}_{\alpha}$ transform of a given function $f(x) . \quad$ (1) Suppose that $f \in$ $C^{\infty}(\mathbb{R})$ is compactly supported. Then $\hat{f}(z)$ is an entire function in the complex plane and there exist real scalars $a$ and $b: a<b$, such that for any positive integer $n$,

$$
|\hat{f}(z)| \leq C_{n}(1+|z|)^{n} \exp (\operatorname{Re} z \operatorname{Im} z \cot \alpha) \cdot \begin{cases}e^{-a \operatorname{Im} z / \sin \alpha} & \text { if } \operatorname{Im} z \geq 0 \\ e^{-b \operatorname{Im} z / \sin \alpha} & \text { if } \operatorname{Im} z \leq 0\end{cases}
$$


Here $C_{n}$ is a positive constant only depending on $n$. (2) Conversely, any entire function satisfying the bounds specified above is the $\mathcal{F}_{\alpha}$ transform of a $C^{\infty}$ function supported on $[a, b]$.

The proof follows readily from the classical Paley-Wiener theorem and Namias' integral representation formula (Theorem 6.1).

The second important property of FrFT is its uncertainty principle.

Recall the classical uncertainty principle in either Fourier analysis or quantum mechanics. Let $\phi(x) \in L^{2}(\mathbb{R})$ be a "wave" function with unit $L^{2}$ norm, and $\hat{\phi}(y)$ its Fourier transform. Then

$$
\operatorname{Var}(\phi) \operatorname{Var}(\hat{\phi}) \geq \frac{1}{4} .
$$

Here the variance (or uncertainty) is defined as

$$
\operatorname{Var}(f):=\int_{-\infty}^{\infty}(x-\bar{x})^{2}|f(x)|^{2} d x, \quad \bar{x}:=\int_{-\infty}^{\infty} x|f(x)|^{2} d x .
$$

That is, as in quantum mechanics, $|f(x)|^{2}$ is treated as an probability density function.

Fix $\alpha \in[0, \pi / 2]$. Denote by $\hat{\phi}_{\alpha}(y)$ the $\mathcal{F}_{\alpha}$ transform of a unit wave function $\phi(x)$. Then we have

Theorem 6.4. (Uncertainty Principle for FrFT) For any $\alpha \in[0, \pi / 2]$,

$$
\operatorname{Var}(\phi) \operatorname{Var}\left(\hat{\phi}_{\alpha}\right) \geq \frac{\sin ^{2} \alpha}{4} .
$$

Proof. As in Fourier analysis and quantum mechanics, the simplest proof is constructed algebraically, instead of directly based on the integral formula.

Define two operators $A=x$ and $B=x \cos \alpha-p \sin \alpha$, where $p=-i d / d x$ is the momentum operator. By Proposition 3.1, it is easy to check that $\mathcal{F}_{\alpha} B \mathcal{F}_{\alpha}^{\dagger}=$ $y$. Therefore, using the inner product notation $\langle\cdot, \cdot\rangle$,

$$
\begin{aligned}
\operatorname{Var}\left(\hat{\phi}_{\alpha}(y)\right) & =\int_{-\infty}^{\infty}(y-\bar{y})^{2}\left|\hat{\phi}_{\alpha}(y)\right|^{2} d y \\
& =\left\langle(y-\bar{y}) \hat{\phi}_{\alpha}(y),(y-\bar{y}) \hat{\phi}_{\alpha}(y)\right\rangle \\
& =\langle(B-\bar{B}) \phi(x),(B-\bar{B}) \phi(x)\rangle \\
& =\operatorname{Var}_{\phi}(B) .
\end{aligned}
$$

That is, the uncertainty of $\hat{\phi}_{\alpha}$ is exactly that of the observable $B$. Thus,

$$
\operatorname{Var}(\phi) \operatorname{Var}\left(\hat{\phi}_{\alpha}\right)=\operatorname{Var}_{\phi}(A) \operatorname{Var}_{\phi}(B) \text {. }
$$

Since

$$
[A, B]=A B-B A=-i \sin \alpha,
$$


the standard quantum computation gives (see for example ${ }^{6,7}$ )

$$
\operatorname{Var}_{\phi}(A) \operatorname{Var}_{\phi}(B) \geq \frac{\sin ^{2} \alpha}{4} .
$$

This completes the proof.

Therefore, the lower bound reaches its maximum in the classical Fourier transform $(\alpha=\pi / 2)$.

We conclude the paper with two remarks on the application potential of FrFT. First, based on the experience in signal and image processing, the author believes that the pure quadratic phase transform has intrinsic meaning for signal analysis. See, for example, Shen and Strang ${ }^{8,9}$ for the role of asymptotically pure phase transforms in wavelets and filters. Second, FrFT can be efficiently implemented in computers based on Fast Fourier Transform (FFT). By the structure of the operator $T_{a, \lambda}$, the only pre-processing and post-processing required for an FFT based implementation are the uniform scaling $s_{\lambda}$ and a multiplication by the quadratic phase factor. But they can be implemented straightforwardly since no matrix-vector multiplications are involved.

\section{Acknowledgments}

I would like to thank Professor Eric D'Hoker in the Physics Department of UCLA for helping me on quantum mechanics, and both Professor Hung Cheng in MIT and Professor Joseph Keller in Stanford for teaching me asymptotic methods. I would also like to dedicate this paper to my dear friend and advisor Gil Strang.

\section{References}

1. V. Namias. The fractional order Fourier transform and its application to quantum mechanics. J. Inst. Maths. Applics., 25:241-265, 1980.

2. A. C. McBride and F. H. Kerr. On Namias's fractional Fourier transforms. IMA J. Appl. Math., 39:159-175, 1987.

3. G. Dattoli, A. Torre, and G. Mazzacurati. An alternative point of view to the theory of fractional Fourier transform. IMA J. Appl. Math., 60:215-224, 1998.

4. C. M. Bender and S. A. Orszag. Advanced Mathematical Methods for Scientists and Engineers. McGrawHill, Inc., 1978.

5. P. M. Morse and H. Feschbach. Methods of Theoretical Physics. McGrawHill, London, 1953.

6. R. Strichartz. A Guide to Distribution Theory and Fourier Transform. CRC Press, Florida, 1993.

7. A. Das and A. C. Mellissinos. Quantum Mechanics - A Modern Introduction. Gordon and Breach Science Publishers, Switzerland, 1986. 
8. J. Shen and G.Strang. Asymptotic analysis of Daubechies polynomials. Proc. Amer. Math. Soc., 124:3819-3833, 1996.

9. J. Shen and G.Strang. Asymptotics of Daubechies filters, scaling functions and wavelets. Appl. Comput. Harmon. Anal., 5:312-331, 1998. 